\title{
Application of GPS on Power System Operation
}

\author{
Chunmei Pei ${ }^{1}$, Huiling Guo ${ }^{2}$, Xiuqing Yang ${ }^{1}, \mathrm{Bin} \mathrm{He}^{1}$, Wei Liu ${ }^{1}$, and Xuemei $\mathrm{Li}^{1}$ \\ ${ }^{1}$ Beijing Vocational College of Electronic Science and Technology, Beijing, China \\ ${ }^{2}$ China University of Mining Technology (Beijing), Beijing, China \\ peichunmei988@163.com
}

\begin{abstract}
Applying GPS positioning and navigation technology to power systems, will realize precise navigation of power equipments, improve power system automation of routine works, and enhance working efficiency. In case of emergency, rapid fixing arrangements can be implemented through monitoring and command platform. With advance communication technology, the realtime video can be transferred to the experts all over the world for remote joint consultation.
\end{abstract}

Keywords: Communication Terminal, GPS Technology, Power Transmission Equipment, Position and Navigation.

\section{Introduction}

After years of development, GPS system has been extensively spread out from mainly military usage to civilian ones. Now more and more GPS terminal, such as PND (Portable Navigation Device), CND (Car Navigation Device), GPS Cell Phone, became popular in people's daily lives. GPS-related applications of various industries have also gradually growing, and many industries have their own GPS applications. At present, most technologies of modern Power Enterprises have met international standards, but the methods of inspection and location are at a lower level, still relying on manpower. The equipments of Power Enterprise are diversiform and widely distributed, so it is a fact that many inspection and repairing staff are not familiar with the equipments' accurate positions. The traditional method is that senior staff lead the way for young people, which is a waste of manpower. Particularly in the accident emergency, the traditional method cannot ensure technical personnel to arrive at the scene in time. GPS positioning and navigation system will replace the traditional method with high-tech satellite navigation, which is an ultimate solution to the current problems.

\section{The Advantage of GPS Positioning and Navigation System in Electrical Power System}

\subsection{Improvement in Efficiency of Daily Operation and Maintenance}

China's territory is vast. The number of electrical power equipments is big, and they're widely distributed. Lots of devices are deployed in remote areas, which brings 
much inconvenience for inspection and checking. It's difficult to find the equipments. The traditional method is to train the new generation of staff by senior ones. This approach is neither scientific nor efficient, especially for large-scale activities (such as the Olympic Games), which need regional cooperation. The GPS positions of all equipments are entered into the map data, so everyone can easily find the destination with a GPS device. Labor cost is saved and the efficiency is significantly improved.

\subsection{Improvement in the Ability of Handling Accidents}

Accidents can not be completely avoided today. In addition to active prevention, a quick solution is particularly important after it happens. Quick and accurate arrival at the scene with a precise navigation device could avoid great loss of the country. Meanwhile, with advanced wireless communication systems, real-time videos of the accident are transmitted to the experts all round the country for remote diagnosis, which can greatly enhance the ability to handle accidents.

\section{The Benefits of GPS Positioning and Navigation System in Electrical Power System}

\subsection{Considerable Economic Benefit}

Power failure could cause national economic disaster. A malfunction may cause economic losses from a few hundred million to more than a billion dollars. (Northeast blackout in United States, 2003, according to the USA and Canada Joint Investigation Team's published report in Dec 5,2003, was the most serious in U.S. history to make a total blackout affecting about 50 million citizens. During the two day power outage, plants shut down and companies stopped businesses caused 4 to 10 billion U.S. dollars losses.) When an emergency occurs, every second can be extremely valuable. GPS positioning and navigation system can realize fast positioning, rapid troubleshooting, and hence avoiding economic losses as much as possible. Meanwhile, the reservation of the guides can be avoided in daily operation and maintenance, even in large cross-regional operations. The labor cost is greatly saved.

\subsection{Large Social Benefits}

Electrical power is closely related to everyone' life in civil society. In any city, power failure is an inconceivable disaster. Several large-scale power outage caused by the accident in history gave local people painful memories. Thus, with GPS positioning and navigation system, the ability of troubleshooting is enhanced for Power Enterprises, which has a very significant impact on people's livelihood and social stability.

\section{The Main Functions of GPS Positioning and Navigation in Electrical Power System}

\subsection{Positioning of Transmission Towers, Substations and Offices}

The locations of transmission towers, substations, offices and so on, are preset in GPS devices, as in Fig. 1. With an electronic map in GPS devices, after positioning by 
GPS satellites, the route can be automatically calculated by directly clicking on the destination. The route from the start point to the destination can be reasonably planned and tracked, as in Fig. 2.

\subsection{Dynamically Addition or Subtraction of Location Information According to Requirement}

The system employs open data structure, which makes it convenient to add or remove location information. When new equipments are added, or some equipment is out of

\section{४> Search Results}

Flood Control Coordinates Navigation

Tower Line Navigation

Substations Location Navigation

Offices Location Navigation

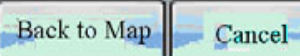

Fig. 1. To preset location information

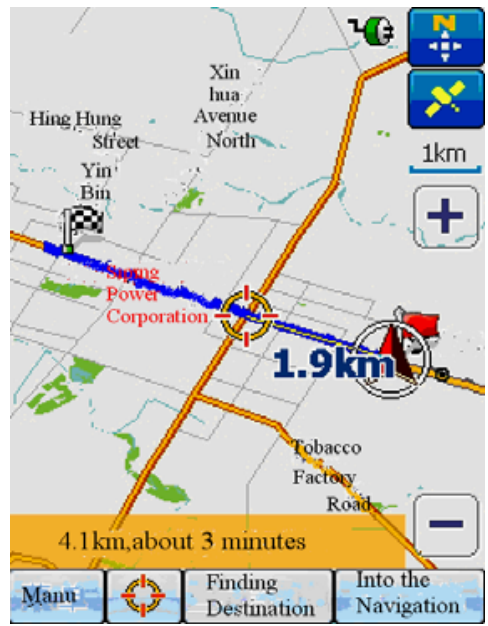

Fig. 2. To start navigation 
date, the user can easily make the change by him/herself. The system also realizes data sharing of address book and navigation path with different devices. Crossregional cooperation can be achieved by simply integrating location information of different areas. New devices are no longer required.

\subsection{Real-Time Monitoring, Improving Management Efficiency}

The GPS navigation device receives GPS satellite signals, automatically positions, and sends the location information in the forms of SMS or data (GPRS / 3G, etc.), to the master control center, via built-in wireless modules, as shown in Fig. 3. The master control center receives the information, extracts the location information, and dynamically displays the longitude, latitude, speed, status, etc., of the vehicles on the electronic map, as shown in Fig. 4. By integrating the data collected, the corporation can find the most appropriate operating fashion, avoid waste, and save cost. By digging deeper into the data, the analyzer can provide the most authentic and reliable reference to the management team, to make more opportunities.

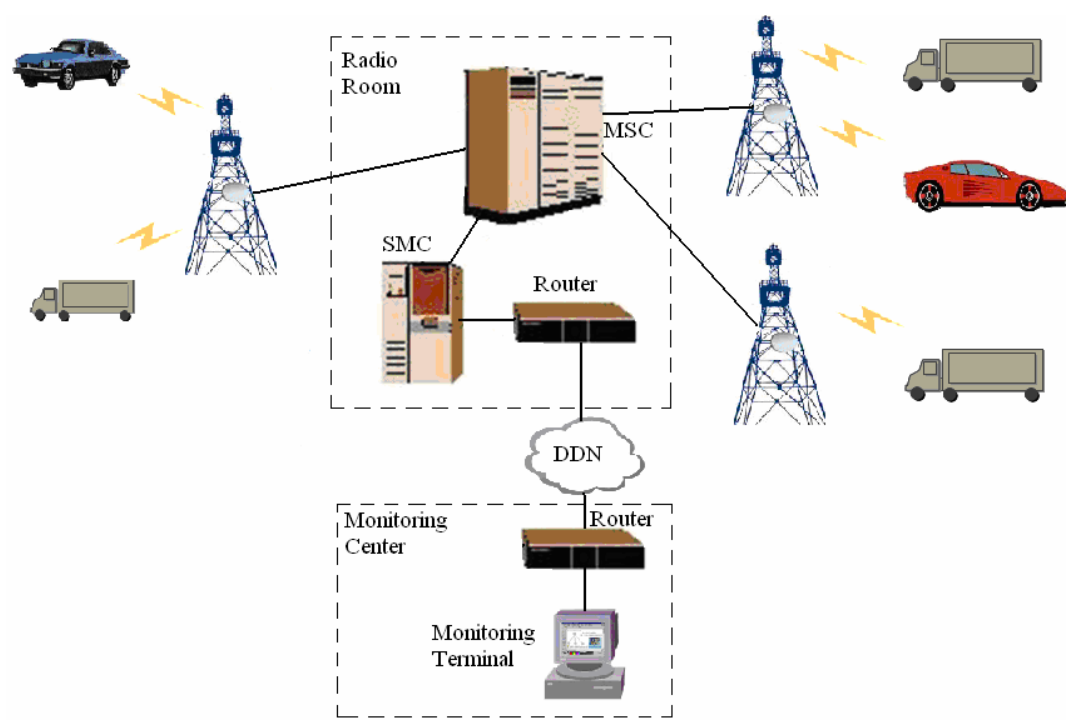

Fig. 3. GPS Monitoring System

\subsection{Combined with Advanced Network, to Enhance Emergency Response Capabilities}

When dealing with urgent accidents, the monitoring platform can accurately obtain the distribution of vehicles and personnel, and carry out overall arrangements. At the same time, GPS positioning and navigation system not only can guide staff to the scene quickly, but can communicate with supervisors via wireless communication capabilities in time, to obtain the correct commands. When facing with complex problems, live scene video can be sent to the master-monitoring center via advanced wireless network (GPRS/3G, etc.). Experts from different regions can participate in the multi-party consultation to diagnose and resolve the problem in time, saving the loss. 


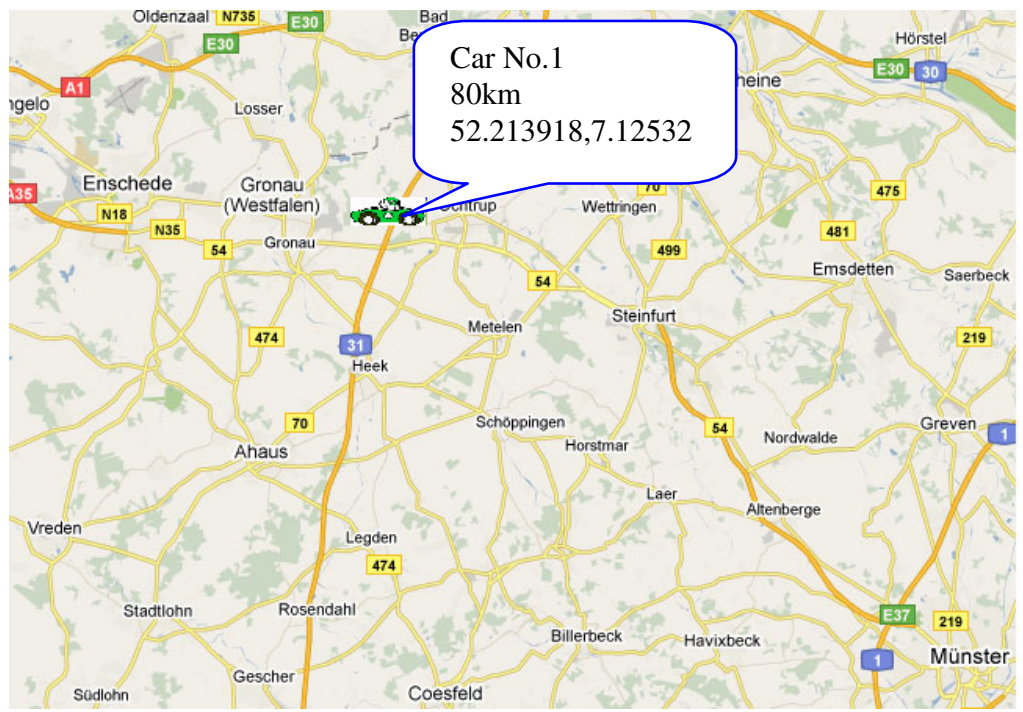

Fig. 4. GPS system monitoring interface sketch

\section{Conclusions}

Electricity is closely related to people's lives. Power Enterprises are taking on more social responsibilities than other general ones. It is Power Enterprises' duty to keep the power grid working stably and regularly. GPS positioning and navigation system, which relies on advanced technologies and is integrated with modern Internet applications, will greatly reform the way of inspection and routine work, thus improving working efficiency. It can also play a valuable role in emergency. As GPS positioning and navigation system is widely used in Power Enterprises, it is no doubt that the whole power grid's modernization and technology level will be improved. It will make a positive contribution to the society.

\section{Reference}

1. Zhu, K.: GPS Application in Power System. J. Computer \& Digital Engineering, Beijing (2007) 